\title{
Influencia del síndrome de Gilbert en los valores de bilirrubina sérica y presencia de litiasis vesicular en pacientes con hemólisis crónica congénita
}

\author{
E. Costa ${ }^{\mathrm{a}}$, R. Pinto ${ }^{\mathrm{b}}$, E. Vieirac ${ }^{\mathrm{c}}$, S. Polo ${ }^{\mathrm{a}}$, A.M. Sarmento ${ }^{\mathrm{a}}$, I. Oliveira ${ }^{\mathrm{a}}$, R. Pimenta ${ }^{\mathrm{d}}$, \\ R. dos Santos ${ }^{\mathrm{C}}$ y J. Barbot ${ }^{\mathrm{a}}$
}

aserviço de Hematologia. Hospital de Crianças Maria Pia. berviço de Pediatria. Centro Hospitalar de Vila Nova de Gaia. 'Unidade de Genética Molecular do Instituto de Genética Médica Doutor Jacinto de Magalhães.

${ }^{\mathrm{d} E s c o l a}$ Superior de Tecnologia de Saúde do Porto. Portugal.

\section{Resumen}

Con el objetivo de evaluar si la presencia de sindrome de Gilbert aumenta el riesgo de desarrollar litiasis vesicular en niños con enfermedad hemolítica crónica, se estudiaron 44 niños con este diagnóstico. El diagnóstico de litiasis vesicular se estableció mediante ultrasonografia. Esta se efectuó anualmente de forma protocolarizada o en el contexto de una sintomatología abdominal dolorosa. En todas ellas, se registraron los valores medios de hemoglobina, reticulocitos y bilirrubina total y directa en fase crónica. Además se analizó la presencia de la inserción del dinucleótida TA en la región promotora del gen de la enzima uridina-difosfoglucuronosiltransferasa (UGT1A1) que está asociada al síndrome de Gilbert.

Encontramos 10 pacientes homozigotos para la inserción dinucleótida $T A * 7 / T A * 7(22,7 \%), 12$ heterozigotos para la inserción dinucleótida $T A * 6 / T A^{*} 7(27,3 \%)$ y 22 pacientes homozigotos para el alelo normal $T A * 6 / T A * 6$ ( $50 \%)$. No se encontraron diferencias estadisticamente significativas en los valores de hemoglobina (test de Kruskal-Wallis $=2,496 ; p>0,05)$ ni en el recuento de reticulocitos (test de Kruskal-Wallis $=1,696 ; p>0,05$ ) en los 3 grupos de pacientes. Esto sugiere un grado similar de hemólisis. La media de la bilirrubina total en los pacientes con el genotipo $T A^{*} 7 / T A^{*}$ fue significativamente superior a la de los que presentaban el genotipo $T A * 6 / T A * 6$ (test de Mann-Whitney $=35,5 ; p<0,05)$. Ningún paciente con el genotipo $T A * 6 / T A * 6$ presentó litiasis vesicular, pero 2 de los 12 con el genotipo $T A * 6 / T A * 7(16,6 \%)$ y 6 de los 10 con el genotipo $T A * 7 / T A * 7(60 \%)$ tuvieron esta complicación.
En este último grupo, 4 pacientes presentaron pancreatitis aguda como complicación de la litiasis vesicular.

La asociación entre la producción de la bilirrubina aumentada debido a una enfermedad hemolítica crónica y la disminución de la conjugación hepática de ésta condiciona un incremento de bilirrubina en la bilis. En consecuencia, esto conlleva a un riesgo aumentado en la formación de litiasis vesicular. Así, en la evolución inicial de un niño con enfermedad hemolítica crónica tendrá importancia la investigación del síndrome de Gilbert.

Palabras clave:

Síndrome de Gilbert. UGT1A1. Bilirrubina. Anemia. Hemólisis. Litiasis vesicular. Pancreatitis aguda.

\section{INFLUENCE OF GILBERT'S SYNDROME ON SERUM BILIRUBIN LEVELS AND GALLSTONE FORMATION IN CHILDREN WITH CHRONIC HEMOLYTIC DISEASE}

To determine whether Gilbert's syndrome increases the risk of gallstone formation in children with chronic hemolytic disease, we studied 44 children with this diagnosis. Gallstones were detected by abdominal ultrasonography. This took place annually in scheduled examinations or in the context of acute abdominal pain. In all patients, the mean values of hemoglobin, reticulocyte and serum bilirubin in the chronic phase were recorded. In addition, TA insertion in the A(TA) ${ }_{n}$ TATAA motif within the promoter region of the enzyme uridine-diphosphate-glucuronyl

Trabajo subvencionado por la Comissão de Fomento da Investigação em Cuidados de Saúde, Ministério da Saúde, Portugal.

Correspondencia: Dr. E. Costa.

Serviço de Hematologia. Hospital de Crianças Maria Pia.

Rua da Boavista, 827. 4050-111 Porto. Portugal

Correo electrónico: hematologia@hmariapia.min-saude.pt elisio_costa@hotmail.com

Recibido en marzo de 2002

Aceptado para su publicación en septiembre de 2002. 
transferase (UGT1A1) was screened, since this is typically associated with GS.

We found $10(22.7 \%)$ homozygotes for the mutated allele $T A * 7 / T A * 7,12(27.3 \%) T A * 6 / T A * 6$ heterozygotes and $22(50 \%)$ homozygotes for the wild-type allele $T A * 6 / T A * \sigma$. No statistically significant differences were found in the values of hemoglobin (Kruskal-Wallis test = 2.496; p $>0.05$ ) or in reticulocyte count (Kruskal-Wallis test $=1.696 ; p>0,05)$ between the three groups of patients, suggesting a similar degree of hemolysis. Patients with the UGT1A1 $T A * 7 / T A * 7$ genotype showed higher mean serum bilirubin levels than did patients who were homozygous for the wild-type allele (Mann-Whitney test $=35.5 ; p<0.05)$. None of the patients with the $T A * 6 / T A * 6$ genotype developed gallstones, whereas this complication was found in 2 of $12(16.6 \%)$ heterozygotes and 6 of $10(60 \%)$ homozygotes for the allele with TA insertion. In this latter group, 4 patients presented acute pancreatitis as a consequence of gallstone formation.

The association between increased bilirubin load due to chronic hemolytic disease and diminished hepatic conjugation leads to raised serum bilirubin levels and consequently to an increased risk of gallstone formation. Therefore, we recommend screening for Gilbert's syndrome in children in the initial phases of chronic hemolytic diseases.

Key words:

Gilbert's syndrome. UGT1A1. Bilirubin. Anemia. Hemolytic disease. Gallstones. Acute pancreatitis.

\section{INTRODUCCIÓN}

La mayoría de las veces, las enfermedades hemolíticas crónicas de carácter congénito, resultan de una enfermedad intrínseca al eritrocito. Esto tiene lugar en el ámbito de su membrana, molécula de hemoglobina o sistema enzimático. La presencia de anemia, de carácter más o menos grave, resulta del equilibrio entre el grado de disminución de la supervivencia eritrocitaria y el de regeneración medular. Los procesos de hemólisis crónica se caracterizan por una hiperbilirrubinemia predominantemente no conjugada, resultado de la destrucción eritrocitaria acelerada ${ }^{1}$. La cantidad de bilirrubina sérica no depende sólo de la cantidad producida, sino también de la capacidad del hepatocito de proceder a su conjugación y excreción. Por tanto, las concentraciones de bilirrubina base en un enfermo con hemólisis crónica dependen del grado de su hemólisis y de su capacidad de conjugar$\mathrm{la}^{1,2}$. Desde el punto de vista clínico este equilibrio es importante, pues una de las complicaciones más frecuente y grave de la enfermedad hemolítica crónica es la formación de cálculos biliares de bilirrubinato resultante de hiperbilirrubinemia ${ }^{2}$. La decisión delicada de realizar esplenectomía en un niño con enfermedad hemolítica crónica depende de diversos factores como: patología eritrocitaria en causa, gravedad de la hiperbilirrubinemia y de la anemia subyacente ${ }^{2}$. Así, la prevención de litiasis ve- sicular puede constituir por sí sola un criterio de esplenectomía en situaciones consideradas de mayor riesgo. En este sentido, será importante la valoración de la capacidad de conjugación de la bilirrubina por parte del hepatocito $^{1,2}$.

El síndrome de Gilbert es una enfermedad común caracterizada por una forma benigna de hiperbilirrubinemia no conjugada en ausencia de disfunción hepática y hemólisis ${ }^{3,4}$. El síndrome se debe a un déficit parcial de la glucuronoconjugación hepática de la bilirrubina, que resulta de una disminución de cerca del $30 \%$ de la actividad de la enzima uridina-difosfoglucuronosiltransferasa (UGT1A1). El déficit grave de esta enzima es responsable del síndrome de Crigler-Najjar. La primera mutación se describió en el gen UGT1A1 en 1992. Se trataba de una mutación nonsense encontrada en un enfermo con síndrome de Crigler-Najjar ${ }^{5}$. Sin embargo, las primeras mutaciones relacionadas con el síndrome de Gilbert fueron descritas en 1995. Una de ellas es una inserción de dos nucleótidos (TA) en la región promotora del gen, que se considera como principal causa de síndrome de Gilbert en todas las poblaciones estudiadas ${ }^{6}$. Esta inserción en la región A(TA)nTAA del promotor del gen UGT1A1 dificulta la unión del factor transcripcional IID, siendo éste importante en el inicio de la transcripción. Esto condiciona una disminución de la actividad de la enzima UGT1A1 y en consecuencia un aumento de los valores de bilirrubina en individuos normales y en enfermos portadores de procesos hematológicos que condicionan hemólisis ${ }^{7-12}$.

El objetivo de este estudio es evaluar si la presencia de un síndrome de Gilbert podrá constituir un factor de riesgo adicional de litiasis vesicular en niños con enfermedad hemolítica crónica.

\section{MATERIAL Y MÉTODOS}

\section{Población de pacientes}

Se estudiaron 44 niños, con una edad media $( \pm \mathrm{DE})$ de 9,7 $\pm 5,1$ años, con el diagnóstico de enfermedad hemolítica crónica (30 casos de esferocitosis hereditaria, seis de déficit de glucosa-6-fosfato deshidrogenasa de la clase I, 6 de déficit de piruvatocinasa, uno de xerocitosis hereditaria y uno sin diagnóstico etiológico). En todos ellos, se registraron los valores medios de hemoglobina, reticulocitos y bilirrubina total. Se excluyeron los valores encontrados en fase aguda. En los niños esplenectomizados sólo se consideraron los valores referentes al período preesplenectomía.

El diagnóstico de litiasis vesicular se estableció por ultrasonografía. Ésta se efectuó anualmente de forma protocolarizada o en el contexto de una sintomatología abdominal dolorosa.

Se solicitó autorización a los responsables de los niños para participar en el estudio, exponiendo el objetivo y metodología de éste. 
Este trabajo fue presentado previamente bajo la forma de anteproyecto a la Comisión de Ética del Hospital Maria Pia, habiendo sido aprobado.

\section{Genotipo de la región A(TA)nTAA del gen UGT1A1}

La región A(TA)nTAA del promotor del gen $U G T 1 A 1$ fue amplificada por reacción en cadena de la polimerasa (PCR). Se utilizaron un par de cebadores descritos por Bancroft et $\mathrm{al}^{13}$, uno de los cuales fue marcado con un fluorocromo. Los productos de amplificación se separaron por electroforesis capilar. Posteriormente, se analizaron en el programa ABI GeneScan (Applied Biosystems) para determinar el tamaño del fragmento y respectivo número de repeticiones TA. Este número se confirmó en dos muestras homozigotas para $T A^{*} 6 y A^{*} 7$ por secuenciación automática.

\section{Análisis estadístico}

El test de Kruskal-Wallis se utilizó para comparar las diferencias entre las edades y los valores de hemoglobina y reticulocitos en los 3 grupos de pacientes (homozigotos normales, heterozigotos y homozigotos para la inserción TA). El test de Mann-Whitney se utilizó en la comparación de los valores medios de bilirrubina total entre los 3 grupos de pacientes. El test de Fisher se usó para comparar la proporción de pacientes que desarrollaron litiasis vesicular. Las diferencias se consideraron significativas cuando $\mathrm{p}<0,05$.

\section{Resultados}

Se encontraron 10 pacientes homozigotos para la inserción dinucleótida $T A^{*} 7 / T A^{*} 7(22,7 \%), 12$ heterozigotos para la inserción dinucleótida $T A^{*} 6 / T A^{*} 7(27,3 \%)$ y $22(50 \%)$ pacientes homozigotos para el alelo normal $T A^{*} 6 / T A^{*} 6$ (fig. 1). La frecuencia de la inserción dinucleótida TA fue de 0,36 en este grupo de pacientes, semejante a la observada en la población general sin enfermedades hemolíticas ${ }^{5,11}$

En el grupo de pacientes homozigotos normales la media ( \pm DE) de hemoglobina fue de 10,03 $\pm 1,62 \mathrm{~g} / \mathrm{dl}$, en los heterozigotos de $10,55 \pm 1,3 \mathrm{~g} / \mathrm{dl}$ y en el de los homozigotos para la inserción TA fue de 10,91 $\pm 1,78 \mathrm{~g} / \mathrm{dl}$. Estos valores no revelan diferencias estadísticamente significativas en los 3 grupos de pacientes $(p>0,05)$. En el grupo de pacientes homozigotos normales la media $( \pm \mathrm{DE})$ del número de reticulocitos fue de $444 \pm 73 \times 10^{9} / 1$, en los heterozigotos de $391 \pm 161 \times 10^{9} / 1$ y en el de los homozigotos para la inserción TA fue de $358 \pm 197 \times 10^{9} / 1$. Tampoco en este parámetro se encontraron diferencias estadísticamente significativas en los 3 grupos ( $p>0,05)$, ni en las edades en los 3 grupos de pacientes ( $p>0,05$ ). Por el contrario, la media de bilirrubina total (fig. 2) en los pacientes con el genotipo $T A^{*} 7 / T A^{*} 7$ fue significativamente superior en los pacientes con genotipo $T A^{*} \delta / T A^{*} \sigma(\mathrm{p}<0,05)$.

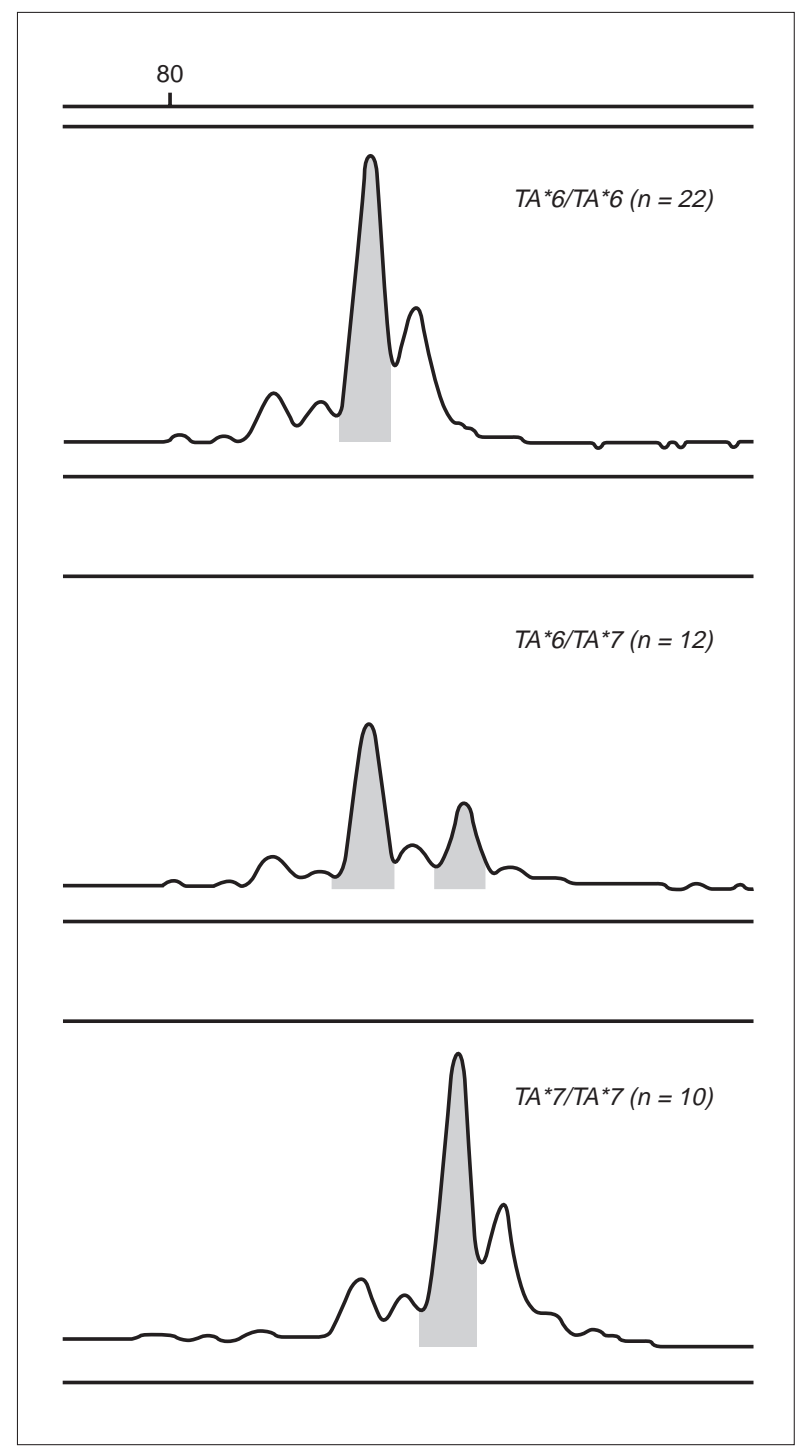

Figura 1. Electrofenogramas del tamaño del fragmento obtenido en el programa ABI GeneScan, correspondientes a los diferentes genotipos encontrados.

En los pacientes con el genotipo $T A * 6 / T A^{*} 7$ no se encontró evidencia estadística ( $\mathrm{p}>0,05)$ de que los niveles de bilirrubina total fuesen superiores a los de los pacientes homozigotos normales. Así, la media $( \pm \mathrm{DE})$ de bilirrubina total fue $T A^{*} \sigma / T A^{*} \sigma: 32,0 \pm 15,42 \mu \mathrm{mol} / 1 ; T A^{*} 6 / T A^{*} 7$ : 55,64 \pm 29,36 $\mu \mathrm{mol} / \mathrm{l} ;$ y TA*7/TA*7: 83,7 \pm 57,43 $\mu \mathrm{mol} / 1$.

Ningún paciente con el genotipo $T A^{*} \delta / T A^{*} 6$ presentó litiasis vesicular. Tuvieron esta complicación 2 de los 12 pacientes con el genotipo $T A^{*} 6 / T A^{*} 7(16,6 \%)$ y 6 de los 10 con el genotipo $T A^{*} 7 / T A^{*} 7(60 \%)$. En este último grupo, 4 pacientes desarrollaron pancreatitis aguda, resultado de la litiasis vesicular. Se encontró una diferencia estadísticamente significativa en la proporción de pacientes que presentaron litiasis vesicular entre el grupo de pacientes homozigotos para la inserción TA y el grupo cons- 


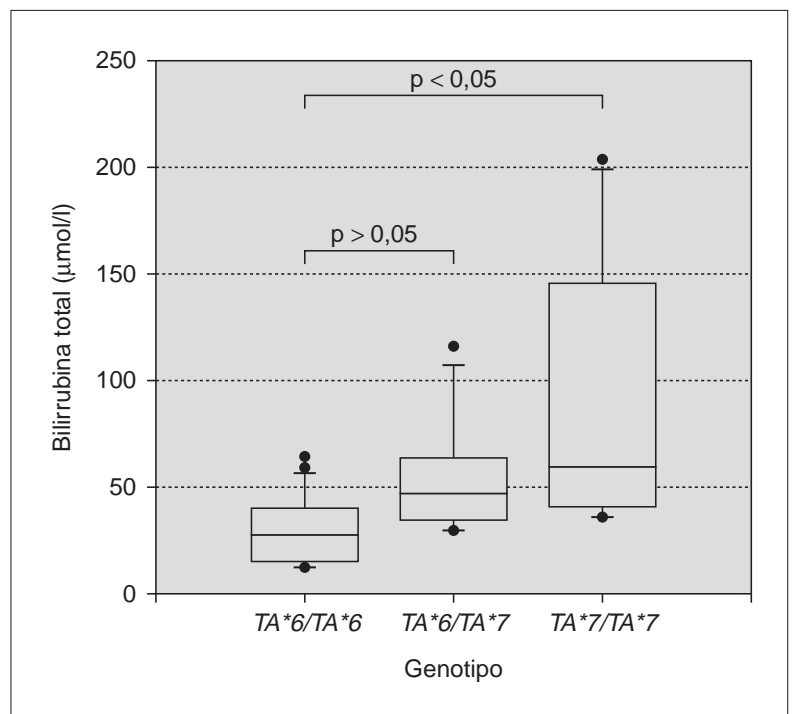

Figura 2. Distribución de los valores de bilirrubina total en los 3 grupos de pacientes. La media de bilirrubina total en los pacientes con el genotipo TA*7/TA*7 fue significativamente superior a los pacientes con genotipo $\mathrm{TA}^{*} 6 / \mathrm{TA}^{*} 6$.

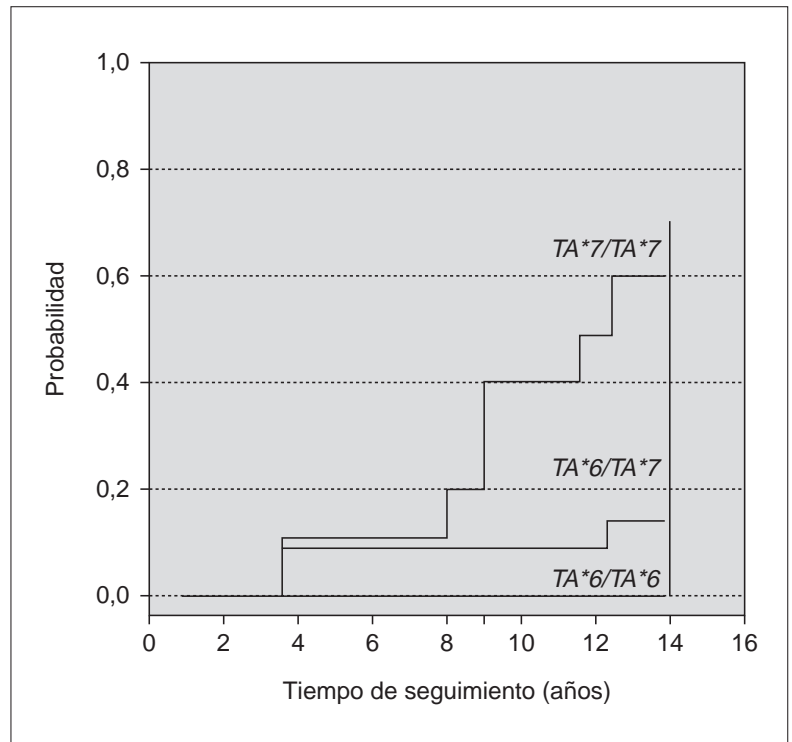

Figura 3. Probabilidad de apariencia de litiasis vesicular (curva de Kaplan Meier) en los 44 pacientes.

tituido por los homozigotos normales y heterozigotos $(\mathrm{p}<0,05)$. En la figura 3 se representan las curvas de probabilidad de supervivencia sin litiasis de los individuos con los diferentes genotipos.

\section{DisCUSIÓN}

La decisión de esplenectomizar a un paciente con enfermedad hemolítica crónica, sobre todo cuando presenta esferocitosis hereditaria, es controvertida. El riesgo aumentado de sepsis grave en pacientes esplenectomizados asumió una relevancia progresiva a lo largo del tiempo. Esta última se acentuó cuando aparecieron estirpes de neumococo resistentes a la penicilina ${ }^{1,2,11,14}$. Como tal, y en particular en niños, se ha realizado un esfuerzo en el sentido de objetivar al máximo sus indicaciones ${ }^{2,11}$, ponderando la relación riesgo-beneficio.

Uno de los beneficios eventuales de la esplenectomía en pacientes con enfermedad hemolítica crónica es la prevención de la litiasis vesicular ${ }^{1,2}$. Así, se realizó un esfuerzo para identificar pacientes con riesgo de presentar esta complicación. Los resultados del presente estudio sugieren que la identificación en el ámbito molecular del síndrome de Gilbert puede dar una consistencia mayor a la delicada decisión de proceder a una esplenectomía. Esto también puede extrapolarse a niños cuyo grado de hemólisis no aparenta ser significativo. Efectivamente, en nuestros 3 grupos de pacientes (homozigotos normales, heterozigotos y homozigotos para la inserción TA) no se encontraron diferencias estadísticamente significativas en las edades y en los valores de hemoglobina y reticulocitos. Ello sugiere un grado similar de hemólisis entre los 3 grupos de pacientes. Encontramos un aumento estadísticamente significativo con relación a los valores de bilirrubina total y a la proporción de pacientes que desarrollaron litiasis vesicular en el grupo homozigoto para la inserción dinucleotídica. En la literatura descubrimos solamente dos trabajos ${ }^{11,15}$ que pretenden determinar si la coexistencia de síndrome de Gilbert y enfermedad hemolítica crónica podrá actuar sinérgicamente aumentando el riesgo de desarrollar litiasis vesicular. En ambos, tal como en este trabajo, se encontró evidencia de riesgo incrementado.

Los 4 casos que presentaron pancreatitis aguda como complicación de la litiasis vesicular plantearon la cuestión de si la asociación de enfermedad hemolítica crónica y síndrome de Gilbert podrá aumentar no sólo el riesgo de litiasis vesicular, sino también la gravedad de sus manifestaciones. Además, se planteó la cuestión de si la esplenectomía pudiese prevenir la litiasis y sus complicaciones, incluso en las situaciones en que la anemia es mínima o está ausente.

Puede concluirse que la asociación entre la producción de bilirrubina aumentada debido a una enfermedad hemolítica crónica y la disminución de la conjugación hepática de ésta condicionan un incremento de bilirrubina en la bilis. En consecuencia, esto conlleva un riesgo aumentado en la formación de litiasis vesicular. Así, en la evolución inicial de un niño con enfermedad hemolítica crónica tendrá importancia la investigación del síndrome de Gilbert. En un futuro, podría ser un criterio de esplenectomía, sobre todo en niños portadores de hemólisis ligera o moderada que presenten ese factor de riesgo. 


\section{BibliografíA}

1. Lee GR, Bithell TC, Foerster J, Athens JW, Lukens JN. Wintrobe's clinical haematology, 9․ㅡㄹ. ed. Philadelphia: Lea and Febiger, 1993.

2. Nathan DG, Orking SH. Nathan and Oski's haematology of infancy and childhood $5^{\text {th }}$ ed. Philadelphia: WB Saunders, 1998.

3. Burchell B, Hume R. Molecular genetic basis of Gilbert's syndrome. J Gastroenterol Hepatol 1999;14:960-6.

4. Maruo Y, Sato H, Yamano T, Doida Y, Shimada M. Gilbert syndrome caused by homozygous missense mutation (Tyr486Asp) of bilirubin UDP-glucuronosyltransferase gene. J Pediatr 1998;132:1045-7.

5. Beutler E, Gelbart T, Demina A. Racial variability in the UDPglucuronosyltransferase 1 (UGT1A1) promoter: A balanced polymorphism for regulation of bilirubin metabolism? Proc Natl Acad Sci USA 1998;95:8170-4.

6. Bosma PJ, Chowdhury JR, Bakker C, Gantla S, De Boer A, Oostra BA, et al. The genetic basis of the reduced expression of bilirubin UDP-glucuronosyltransferase 1 in Gilbert's syndrome. N Engl J Med 1995;333:1171-5

7. Kaplan M, Hammarman C, Renbaum P, Klein G, Levy-Lahad E. Gilbert's syndrome and hyperbilirubinaemia in ABO-incompatible. Lancet 2000;356:652-3.

8. Kaplan M, Beutler E, Vreman HJ, Harmmerman C, Levy-Lahad E, Renbaum P, et al. Neonatal hyperbilirubinemia in glucose-6-phosphate dehydrogenase deficient heterozygotes. Pediatrics 1999; 104:68-74
9. Iolascon A, Faienza MF, Giordani L, Perrotta S, Ruggiu G, Meloni GF, et al. Bilirubin levels in the acute hemolytic crisis of G6PD deficiency are related to Gilbert's syndrome. Eur J Haematol 1999;62:307-10.

10. Galanello R, Perseu L, Melis MA, Cipollina L, Barella S, Giagu $\mathrm{N}$, et al. Hyperbilirubinaemia in heterozygous beta-thalassaemia is related to co-inherited Gilbert's syndrome. Br J Haematol 1997:99:433-6.

11. Giudice EM, Perrota S, Nobili B, Specchia C, d'Urzo G, Iolascon A. Coinheritance of Gilbert syndrome increases the risk for developing gallstones in patients with hereditary spherocytosis. Blood 1999;94:2259-62.

12. Alexandrino AM, Carvalho C, Costa E, Vieira E, Oliveira P, Duarte C, et al. TATA box polymorphism in the UDP-glucoronosyltransferase 1 gene promoter and neonatal hyperbilirubinemia. Prenat Neonat Med 2001;6:133-6.

13. Bancroft JD, Kreamer B, Gourley GR. Gilbert syndrome accelerates development of neonatal jaundice. J Pediatr 1998;132 656-60

14. Schilling RF. Spherocytosis, splenectomy, strokes, and heart attacks. Lancet 1997;350:1677-8.

15. Passon RG, Howard TA, Zimmerman SA, Schultz WH, Ware RE. Influence of bilirubin uridine diphosphate glucuronosyltransferase $1 \mathrm{~A}$ promoter polymorphisms on serum bilirubin levels and colelithiasis in children with sickle cell anemia. J Pediatr Hematol Oncol 2001;23:448-51 